1 From collocations to call-ocations: using linguistic methods to quantify animal

2

3

$4 \quad$ Alexandra B. Bosshard ${ }^{1,2^{*}}$, Maël Leroux ${ }^{1,2}$, Nicholas A. Lester $^{1,2}$, Balthasar Bickel ${ }^{1,2}$, Sabine 5

6

${ }^{1}$ Department of Comparative Language Science, University of Zurich, Switzerland

${ }^{2}$ Center for the Interdisciplinary Study of Language Evolution (ISLE), University of Zurich

${ }^{3}$ Department of Psychology, University of Warwick, UK

${ }^{*}$ Corresponding author:

A. Bosshard, Department of Comparative Language Science, University of Zurich, Thurgauerstrasse 30, 8050 Zurich, Switzerland.

\section{call combinations}




\section{Abstract:}

19 Emerging data in a range of non-human animal species have highlighted a latent ability to

20 combine certain pre-existing calls together into larger structures. Currently, however, there

21 exists no objective quantification of call combinations. This is problematic because animal

22 calls can co-occur with one another simply through chance alone. One common approach

23 applied in language sciences to identify recurrent word combinations is collocation analysis.

24 Through comparing the co-occurrence of two words with how each word combines with

25 other words within a corpus, collocation analysis can highlight above chance, two-word

26 combinations. Here, we demonstrate how this approach can also be applied to non-human

27 animal communication systems by implementing it on a pseudo dataset. We argue

28 collocation analysis represents a promising tool for identifying non-random,

29 communicatively relevant call combinations in animals.

31 Key-words: call combinations, collocation analysis, comparative approach, non-random

32 structure, syntax 


\section{Introduction}

35 Over the last 20 years there has been growing interest into the combinatorial abilities of animals, namely the propensity to sequence context-specific calls (i.e. meaning-bearing

37 units, see (Suzuki \& Zuberbühler, 2019) into larger potentially meaningful structures (Arnold

38 \& Zuberbühler, 2006; Collier et al., 2020; Engesser, Ridley, \& Townsend, 2016; Ouattara,

39 Lemasson, \& Zuberbühler, 2009; Suzuki, Wheatcroft, \& Griesser, 2016). Combinatoriality is

40 one mechanism that can increase the expressive potential of a finite vocal repertoire. It

41 therefore provides important comparative insights into the complexity of animal vocal

42 systems and the selective pressures such systems have been exposed to (Collier et al.,

43 2020). These data also hold great promise in furthering our understanding of the similarities

44 between animal communication and human language given that, for many years, it was assumed that the systematic concatenation of meaning-bearing units (i.e., syntax) was a phenomenon unique to language (Hurford, 2012). Emerging examples of syntactic-like structure in non-human primates and non-primate animals suggests this particular assumption was indeed premature (Arnold \& Zuberbühler, 2006; Berthet et al., 2019; Coye, Ouattara, Zuberbühler, \& Lemasson, 2015; Coye, Zuberbühler, \& Lemasson, 2016) and such data even have the potential to further our understanding of the evolutionary progression of our own communication system (Leroux \& Townsend, 2020; Townsend, Engesser, Stoll,

52 Zuberbühler, \& Bickel, 2018).

54 In light of the communicative and evolutionary insights that research on combinatoriality can provide, it is surprising that, to date, no objective means of quantifying call combinations has been proposed. This is problematic as animal calls may occur in rapid 
method to capture greater-than-chance co-occurrence of calls is therefore central to reliably call combinations.

Similar methodological issues have been encountered in research on language learning and use (Bartsch, 2004; Evert, 2008; Gablasova, Brezina, \& McEnery, 2017; Gries, 2013). One approach frequently implemented to identify combinations of words (mostly bigrams i.e., two-word/two-call structures) in large written and spoken corpora is collocation analysis (for a review, see Gries, 2013). Collocation analyses can take several forms, but the core commonality is that it contrasts the frequency with which specific words combine to measure the relative exclusivity of their relationship within a corpus (Church, Gale, Hanks, \& Hindle, 1991; Gries \& Stefanowitsch, 2004; Kennedy, 1991; Nesselhauf, 2005). In other words, such analyses reveal whether particular word/call combinations are more common than would be expected given an assumed random baseline (e.g., the uniform distribution, in which each combination is equally likely). For example, in English "drink" collocates with "coffee" and "going" collocates with "to" (to form the future tense or describe a motion event). Thus, collocation analyses can be understood as statistical measures of the influence that a lexical item has on its neighbours. It has since become a crucial tool in Corpus Linguistics for analysing lexical items and grammatical features of natural language (e.g., Bartsch, 2004; Lehecka, 2015; Stefanowitsch \& Gries, 2003; Xiao \& Mcenery, 2006).

In this paper we propose that by considering animal vocal data in a similar way as to how language data are treated (i.e., as a corpus) affords the unique opportunity to apply a 
questions. Specifically, we demonstrate the application of collocation analyses to non-

human animal datasets as a way to empirically identify combinations of two calls,

henceforth termed bigrams, and the relative merits of doing so. We focus on two specific

forms of collocation analysis commonly implemented in language sciences: Multiple

Distinctive and Mutual Information Collocation Analyses (Gries, 2014).

\section{Collocation analyses - Multiple Distinctive and Mutual Information approaches:}

Multiple Distinctive Collocation Analysis (MDCA) is primarily used when investigating and

testing for the degree of attraction between meaning-bearing units that share semantic

similarities in grammatical constructions. Specifically, MDCA statistically contrasts all

possible bigram combinations to estimate whether a given bigram occurs at frequencies

higher or lower than what would be expected by chance (Gries \& Stefanowitsch, 2004;

Hilpert, 2006). Furthermore, the output of MDCA also provides a superficial estimate of

bigram ordering, namely whether the combination is sensitive to the position of the calls

Stefanowitsch, 2004; Hilpert, 2006).

One recurrent issue for the analysis of linguistic corpora is the fact that any corpus

represents an incomplete - or undersampled - representation of the target linguistic system

- i.e., some two-word combinations can be under-represented or even absent from a 
107 however actually overestimates to low-frequency values (Church \& Hanks, 1990; Evert, 2005), which can be an advantage in animal communication corpora, as low-frequency potential combinatorial candidates. MICA calculates the variability of co-occurring items

111 through computing information values via observed frequency divided by expected

112 frequency. MICA can therefore - to some degree - better account for low-frequency

113 bigrams, a common occurrence in small corpora and particularly in non-human vocal

114 datasets.

115 In the remainder of the paper, we apply both forms of collocation analyses using an existing

116 R script provided by Stefan Gries (2014, see supplementary) to a pseudo data set we built

117 for the "Yeti" - a mythical ape-like creature.

119 Call combinations in Yetis: an example:

120 We created an artificial set of call combinations produced by the Yeti (see Table 1). The data

121 set was generated with a range of distributions in mind: specifically call combinations with

122 i) high-frequencies, ii) low frequencies, and call types that appear in combination with a)

123 only one other call type or b) with many different call types. The repertoire and sample size

124 were simulated consistent with the known communicative repertoires of other primates

125 (Leroux et al., in press). Yetis have a call repertoire comprising 10 calls, ranging from tonal

126 whistles and twitters to noisy barks and coughs. Initial assessment of the data suggested

127 that some of the calls co-occur with others from the repertoire and some co-occurrences are more frequent than others. We therefore expected the collocation analyses to reveal at 

the MDCA and MICA to our data set.

131

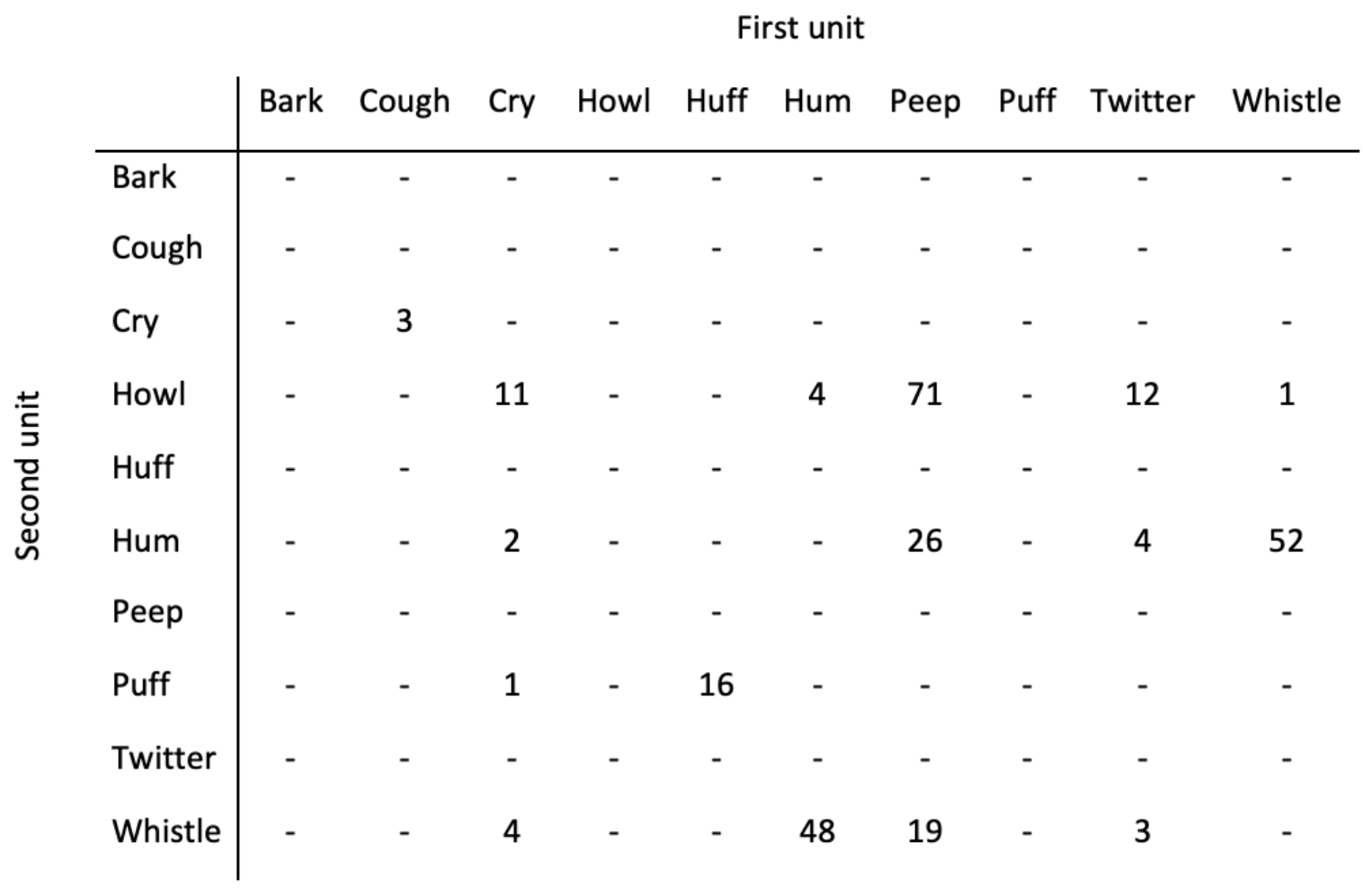

Multiple Distinctive Collocation Analysis:

137 Sixteen different potential bigrams were identified in the Yeti repertoire (see Table 1).

138 In a first step we applied a Multiple Distinctive Collocation Analysis where call dependencies

139 within these bigrams were calculated using an exact binomial test on each possible bigram

140 combination (Gries, 2014; Gries \& Stefanowitsch, 2004; Hilpert, 2006). Specifically, by

141 applying a logarithmic transformation to $p$-values (used here explicitly since they reflect the

142 relative association of calls but whilst simultaneously accounting for sample size), it is then possible to estimate whether a given bigram occurs at frequencies higher (positive "pbins", 
144 Table 2) or lower (negative "pbins", Table 2) than what would be expected by chance (i.e.

145 the absolute value of pbin $\left.>3: \mathrm{P}<0.001,{ }^{*}>2: \mathrm{P}<0.01,{ }^{*}>1.3: \mathrm{P}<0.05\right)$. Since the aim here is to

146 identify potential candidates for meaningful call combinations, we will focus only on positive

147 values that highlight an attraction between two call types. For positive pbin values, the

148 higher the value for two calls, the greater their collocational strength.

149 The Multiple Distinctive Collocation Analysis applied here suggests a significant relative

150 attraction exists within six bigrams (see Table 2). The highest value was calculated for Huff-

151 Puff, followed by Hum-Whistle, Whistle-Hum, Peep-Howl, Cough-Cry and lastly Twitter-

152 Howl. Importantly, four of the six bigrams showed significant attraction between the two

153 comprising calls in one specific order only (Huff-Puff, Peep-Howl, Cough-Cry \& Twitter-Howl,

154 Table 2). However, the combinations involving Hums and Whistles displayed significant

155 attraction no matter the linearisation (Hum-Whistle and Whistle-Hum), suggesting that,

156 either order did not matter for this particular combination, or that Whistle and Hum form

157 two significant, differently ordered bigrams.

158

159 Table 2: Multiple Distinctive Collocation analysis for the bigrams in the Yeti's vocal data set. Columns and rows show the

160 first and second unit within a call combination respectively. Values are pbins and can be translated to p-values (abs(pbin) 


\begin{tabular}{|c|c|c|c|c|c|c|c|c|c|c|}
\hline & \multicolumn{10}{|c|}{ First unit } \\
\hline & Bark & Cough & Cry & Howl & Huff & Hum & Peep & Puff & Twitter & Whistle \\
\hline Bark & - & - & - & - & - & - & - & - & - & - \\
\hline Cough & - & - & - & - & - & - & - & - & - & - \\
\hline Cry & - & 5.9 & -0.1 & - & -0.1 & -0.3 & -0.7 & - & -0.1 & -0.3 \\
\hline Howl & - & -0.5 & 1.2 & - & -2.6 & -4.8 & 8.8 & - & 1.4 & -7.7 \\
\hline Huff & - & - & - & - & - & - & - & - & - & - \\
\hline Hum & - & -0.4 & -1.1 & - & -2.2 & -7.6 & -1.6 & - & -0.5 & 17.0 \\
\hline Peep & - & - & - & - & - & - & - & - & - & - \\
\hline Puff & - & -0.1 & -0.2 & - & 18.6 & -1.5 & -4.0 & - & -0.5 & -1.6 \\
\hline Twitter & - & - & - & - & - & - & - & - & - & - \\
\hline Whistle & - & -0.4 & -0.3 & - & -1.9 & 17.3 & -2.6 & - & -0.6 & -6.8 \\
\hline
\end{tabular}

163

164 In light of the additional aforementioned advantages associated with an information-based approach, we complemented the MDCA with a MICA.

Mutual Information Collocation Analysis:

168 Similarly to MDCA, MICA calculates the collocational strength of each specific call type with every other call type it collocates with. To do so, the joint observed frequency of a specific

170 bigram is divided by its joint expected frequency and then logarithmically transformed.

171 Concretely, the number of times the calls actually appear in combination is divided by the

172 number of times the calls would appear in combination if every call was randomly

173 distributed throughout the dataset. Once more, the higher the collocation value, the

174 stronger the collocational strength between two units (again, we focus on positive values

175 that indicate an attraction only). As with MDCA, pbins represent the logarithmically

176 transformed $p$-values (i.e., the absolute value of pbin $>3$ : $P<0.001,{ }^{*}>2$ : $P<0.01,{ }^{*}>1.3$ :

$177 \quad \mathrm{P}<0.05)$. 
178 Not accounting for any specific ordering of the structures in the received input, the Mutual

179 Information Collocation Analysis demonstrated a significant relative attraction within two

180 bigrams (Cough-Cry \& Huff-Puff, see Table 3). MICA only highlighted bigrams with call types

181 that appear exclusively in combination with their collocational partner and not many other

182 call types, while not rendering significant values for combinations of call types that appear

183 with more than one other call type in the corpus (see Table 1). It is also noteworthy that the

184 MICA provides a strong value for a very low-frequency pairing, namely Cough-Cry, which can

185 only be found three times in the recorded repertoire.

186

187 Table 3: Mutual Information Collocation analysis for the bigrams in the Yeti's vocal data set. Columns and rows show the

188 first and second unit within a call combination respectively. Values are pbins and can be translated to $p$-values (pbin *>3:

$189 \mathrm{P}<0.001, *>2: \mathrm{P}<0.01, *>1.3: \mathrm{P}<0.05)$. Significant results are coloured in green.

190

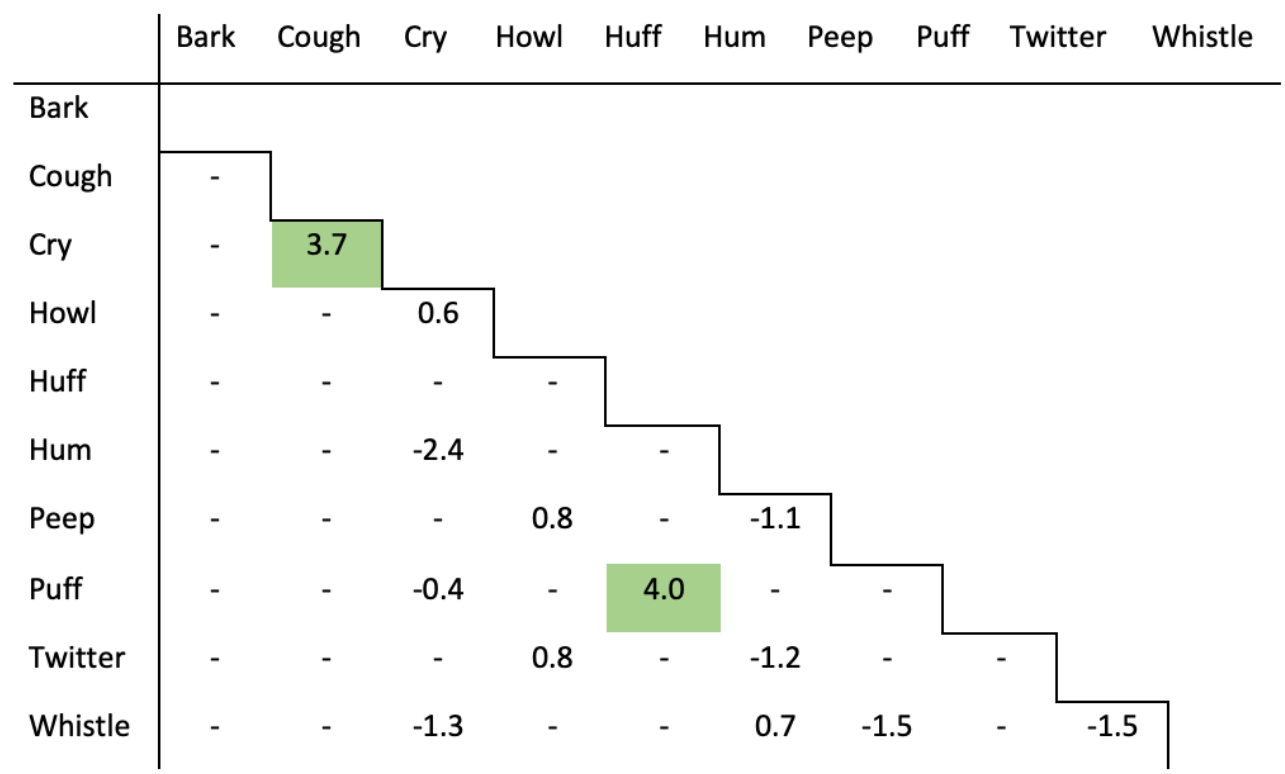

191

Discussion

193 Here, we show that, when conceptualising animal vocal data in the same way as a language corpus, methods habitually implemented in corpus linguistics can be transferred reliably to 
195

196

197

198

199

200

201

202

203

204

205

206

207

208

209

210

211

212

213

214

215

216

217 less clear to us during observational data collection. Firth, 1957).

non-human communication systems to highlight promising call combinations. We argue that

this approach therefore represents a novel application of a more objective method to quantify the combinatorial dynamics of animal communication systems. Specifically, collocation analyses help disentangle "true", or non-random, call combinations from happenstance juxtapositions of single calls. This is critical when investigating potentially meaningful structuring within animal vocal communication systems.

It is important to note that other systematic approaches to capture the sequential dynamics of animal vocal sequences (e.g., song), have been applied, including different Markovian and non-Markovian chain modelling (Kershenbaum et al., 2014; Sainburg, Theilman, Thielk, \& Gentner, 2019; Suzuki, Buck, \& Tyack, 2006). Nevertheless, we argue that there are strong advantages for using collocation analysis, since collocations are easily operationalisable across systems and provide a convenient and more descriptive (as opposed to modellingbased) account of the combinatorial dependencies between vocal units (Evert, 2005, 2008;

Whilst here we implement these analyses only with a pseudo data set, we have also applied MDCA and MICA in real-world settings, primarily when investigating the combinatorial properties of wild chimpanzee (Leroux et al., in press) and captive marmoset (Bosshard, 2020) vocal data sets. In these two primate examples, collocation analyses also reliably identified non-random combinations and, critically, these included ones we had already anecdotally highlighted as promising candidates in addition to others that were previously 
219 Of particular relevance is the fact that the type of collocational analyses applied here were

220 sensitive to bigrams even when they occurred very infrequently in the data set. This is

221 because collocational analyses consider the exclusivity of the combinatorial relationship: if

222 calls combine extremely rarely, they will still be detected as long as their relationship

223 together is exclusive. Since considerable variation characterises the frequency of call

224 combinations in animal communication (e.g., alarm call combinations are less frequent than

225 social call combinations (Boesch \& Crockford, 2005; Collier, Townsend, \& Manser, 2017;

226 Leroux, Chandia, Bosshard, Zuberbühler, \& Townsend, in prep.), we can be confident that

227 collocational analyses will identify all relevant combinations, both common and rare.

228

229 Another advantage of such collocation analyses is that they allow an estimate of the

230 ordering of call combinations. Identifying variation in the temporal progression of calls is necessary to design experiments probing the role of order on meaning: data which are key to unpacking how similar animal call combinations and human language really are. Our results from the provided Yeti dataset indicate that some, but not all, of the identified combinations are characterised by ordering, again a finding that is replicated in our realworld data sets (Bosshard, 2020; Leroux et al., in press). This preliminary identification of call order therefore might serve as one possible additional filter when deciding which of the combinations detected from an animal data set to follow-up from an experimental/playback perspective.

In conclusion, we hope that the approach outlined here will be applied by other researchers in the field of animal communication as a way to disambiguate random from non-random combinatorial structures. Future work could also build on these initial approaches through 
243 applying other, as yet unexplored, sequence-based modelling methods currently used in

244 language sciences to animal corpora (e.g., skip-gram modelling, see Guthrie, Allison, Liu,

245 Guthrie, \& Wilks, 2006). Ultimately, implementing the same objective, standardised

246 methods such as that presented in this paper could allow researchers to make more

247 meaningful comparisons both within and across systems, for example, at the individual,

248 group, population or even species level.

251 Acknowledgments:

252 We thank members of the Comparative Communication and Cognition Group at the

253 University of Zurich, specifically Piera Filippi, Mélissa Berthet, Joseph Mine, Anna J.

254 Szmarowska and Manuel Rüdisühli for discussions. This work was supported by the Swiss

255 National Science Foundation (PPOOP3_163850) to S.W.T. and the NCCR Evolving Language

256 (Swiss National Science Foundation Agreement \#51NF40_180888). We declare no conflict of

257 interest. 


\section{References}

260

261

262

263

264

265

266

267

268

269

270

271

272

273

274

275

276

277

278

279

280

Arnold, K., \& Zuberbühler, K. (2006). Semantic combinations in primate calls. Nature, 441(7091), 303-303.

Bartsch, S. (2004). Structural and functional properties of collocations in English: A corpus study of lexical and pragmatic constraints on lexical co-occurrence. Gunter Narr Verlag.

Berthet, M., Mesbahi, G., Pajot, A., Cäsar, C., Neumann, C., \& Zuberbühler, K. (2019). Titi monkeys combine alarm calls to create probabilistic meaning. Science Advances, 5(5), eaav3991.

Boesch, C., \& Crockford, C. (2005). Call combinations in wild chimpanzees. Behaviour, 142(4), 397-421.

Bosshard, A. B. (2020). Sequential dynamics in common marmoset vocal strings. University of Zurich.

Church, K., Gale, W., Hanks, P., \& Hindle, D. (1991). Using statistics in lexical analysis. Lexical Acquisition: Exploiting on-Line Resources to Build a Lexicon, 115, 164.

Church, K., \& Hanks, P. (1990). Word association norms, mutual information, and lexicography. Computational Linguistics, 16(1), 22-29.

Collier, K., Radford, A. N., Stoll, S., Watson, S. K., Manser, M. B., Bickel, B., \& Townsend, S. W. (2020). Dwarf mongoose alarm calls: Investigating a complex non-human animal call. Proceedings of the Royal Society B, 287(1935), 20192514.

Collier, K., Townsend, S. W., \& Manser, M. B. (2017). Call concatenation in wild meerkats. Animal Behaviour, 134, 257-269. 
Coye, C., Ouattara, K., Zuberbühler, K., \& Lemasson, A. (2015). Suffixation influences receivers' behaviour in non-human primates. Proceedings of the Royal Society B: Biological Sciences, 282(1807), 20150265.

284

Coye, C., Zuberbühler, K., \& Lemasson, A. (2016). Morphologically structured vocalizations in female Diana monkeys. Animal Behaviour, 115, 97-105.

286

Engesser, S., Ridley, A. R., \& Townsend, S. W. (2016). Meaningful call combinations and compositional processing in the southern pied babbler. Proceedings of the National Academy of Sciences, 113(21), 5976-5981.

Evert, S. (2005). The statistics of word cooccurrences: Word pairs and collocations.

Evert, S. (2008). Corpora and collocations. Corpus Linguistics. An International Handbook, 2, $1212-1248$.

Firth, J. R. (1957). Modes of meaning Papers in linguistics 1934-1951 (pp. 190-215). London, England: Oxford University Press.

Gablasova, D., Brezina, V., \& McEnery, T. (2017). Collocations in corpus-based language learning research: Identifying, comparing, and interpreting the evidence. Language Learning, 67(S1), 155-179.

Gries, S. T. (2013). 50-something years of work on collocations: What is or should be next.... International Journal of Corpus Linguistics, 18(1), 137-166.

Gries, S. T. (2014). Coll.analysis 3.5. A script for $R$ to compute perform collostructional analyses.

Gries, S. T., \& Stefanowitsch, A. (2004). Extending collostructional analysis: A corpus-based perspective onalternations'. International Journal of Corpus Linguistics, 9(1), 97-129. 
Hilpert, M. (2006). Distinctive collexeme analysis and diachrony.

Hurford, J. R. (2012). The origins of grammar: Language in the light of evolution II (Vol. 2). Oxford University Press.

Kennedy, G. (1991). Between and through: The company they keep and the functions they serve. na.

Kershenbaum, A., Bowles, A. E., Freeberg, T. M., Jin, D. Z., Lameira, A. R., \& Bohn, K. (2014). Animal vocal sequences: Not the Markov chains we thought they were. Proceedings of the Royal Society B: Biological Sciences, 281(1792), 20141370.

Lehecka, T. (2015). Collocation and colligation. In Handbook of pragmatics online. Benjamins.

Leroux, M., Bosshard, A. B., Chandia, B., Manser, A., Zuberbühler, K., \& Townsend, S. W. (in press). Chimpanzees combine pant-hoots with food-calls into larger structures. Animal Behaviour.

Leroux, M., Chandia, B., Bosshard, A. B., Zuberbühler, K., \& Townsend, S. W. (in prep.). Call combinations in chimpanzees: A social tool?

Leroux, M., \& Townsend, S. W. (2020). Call combinations in great apes and the evolution of syntax. Animal Behavior and Cognition, 7(2), 131-139.

Nesselhauf, N. (2005). Collocations in a learner corpus (Vol. 14). John Benjamins Publishing.

323 Ouattara, K., Lemasson, A., \& Zuberbühler, K. (2009). Campbell's monkeys concatenate vocalizations into context-specific call sequences. Proceedings of the National Academy of Sciences, 106(51), 22026-22031.

Sainburg, T., Theilman, B., Thielk, M., \& Gentner, T. Q. (2019). Parallels in the sequential 
Stefanowitsch, A., \& Gries, S. T. (2003). Collostructions: Investigating the interaction of words and constructions. International Journal of Corpus Linguistics, 8(2), 209-243.

Suzuki, R., Buck, J. R., \& Tyack, P. L. (2006). Information entropy of humpback whale songs. The Journal of the Acoustical Society of America, 119(3), 1849-1866.

332 Suzuki, T. N., Wheatcroft, D., \& Griesser, M. (2016). Experimental evidence for compositional syntax in bird calls. Nature Communications, 7(1), 1-7.

334 Suzuki, T. N., \& Zuberbühler, K. (2019). Animal syntax. Current Biology, 29(14), R669-R671.

335 Townsend, S. W., Engesser, S., Stoll, S., Zuberbühler, K., \& Bickel, B. (2018). Compositionality in animals and humans. PLoS Biology, 16(8), e2006425.

337 Xiao, Richard, \& Mcenery, Tony. (2006). Collocation, Semantic Prosody, and NearSynonymy: A Cross-Linguistic Perspective. Applied Linguistics, 27(1), 103-129. 